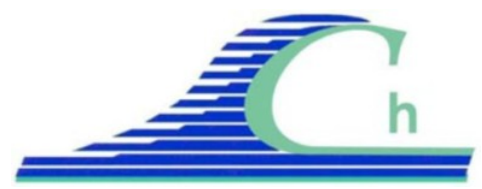

XII ${ }^{\text {imes }}$ Journées Nationales Génie Côtier-Génie Civil

Cherbourg, 12-14 juin 2012

DOI:10.5150/jngcgc.2012.009-K C Editions Paralia CFL

disponible en ligne - http://www.paralia.fr - available online

\title{
Evaluation de profils de viscosité turbulente en couches limites oscillantes par un modèle à deux équations $k-\omega$
}

\author{
Loreline KERLIDOU ${ }^{1}$, Alicia ANDRE ${ }^{1}$, Rafik ABSI ${ }^{1}$, Hitoshi TANAKA ${ }^{2}$
}

1. EBI, 32 Boulevard du Port, 95094 Cergy-Pontoise cedex France.

l.kerlidou@ebi-edu.com; a.andre@ebi-edu.com;r.absi@ebi-edu.com

2. Dept. Civil Eng., Tohoku University, 6-6-06 Aoba, Sendai 980-8579, Japan.

tanaka@tsunami2.civil.tohoku.ac.jp

\section{Résumé :}

La modélisation du transport sédimentaire côtier nécessite la connaissance de la viscosité turbulente en couche limite. Deux principaux modèles analytiques de viscosité turbulente sont utilisés en génie côtier : le profil parabolique-uniforme (VAN RIJN 1993 ; LIU \& SATO, 2006) et le profil exponentiel-linéaire (HSU \& JAN, 1998 ; ABSI 2010). Notre étude a pour objectif d'évaluer et de valider ces deux profils par comparaison avec les résultats d'un modèle de couche limite turbulente à deux équations $k-\omega$. L'étude montre que les deux profils sont possibles en pratique et que l'allure de la viscosité turbulente dépend du paramètre adimensionnel $\alpha_{m} / k_{s}$, où $\alpha_{m}$ est l'amplitude de l'écoulement oscillant et $k_{*}$ la rugosité équivalente de Nikuradse. Le profil parabolique-uniforme est valable pour $a_{m} / k_{s} \geq 500$, tandis que le profil exponentiel-linéaire est adéquat pour $a_{m} / k_{g} \alpha_{n} 500$. Nous proposons des expressions pour les coefficients, qui interviennent dans les modèles analytiques de viscosité turbulente, à partir des résultats du modèle à deux équations $k-\omega$.

\section{Mots-clés :}

Profils de viscosité turbulente - Modèle à deux équations $k-\omega-$ Calibration

\section{Abstract:}

Coastal sediment transport modelling needs the eddy viscosity in the boundary layer. Two analytical eddy viscosity models are largely used in coastal engineering: the parabolic-uniform profile (VAN RIJN, 1993; LIU \& SATO, 2006) and the exponentiallinear profile (HSU \& JAN, 1998 ; ABSI, 2010). The aim of our study is to assess and validate these two profiles by comparison with the results of a turbulent boundary layer two equation $k-\omega$ model. The study shows that the two profiles are possible in practice and the shape of eddy viscosity depends on the dimensionless parameter $a_{m} / k_{g}$, where $a_{m}$ is the wave orbital amplitude and $k_{s}$ the equivalent roughness. The parabolicuniform profile is adequate for $a_{m} / k_{g} \geq 500$, while the exponential-linear profile is more appropriate for $a_{p q} / k_{a}, 500$. We suggest equations for the coefficients of the analytical eddy viscosity models from the results of the two equation $k-\omega$ model.

\section{Keywords:}

Eddy viscosity profiles $-k$ - $\omega$ two equation model - Calibration 


\section{Introduction}

Le transport sédimentaire côtier, qui provoque l'évolution de la ligne de côte et les changements des fonds marins, est dû principalement aux houles et courants (FREDSOE \& DEIGAARD, 1992; VAN RIJN, 1993). L'écoulement oscillant turbulent, que produit la houle au voisinage du fond marin constitué de sédiments, génèrent des structures turbulentes qui déstabilisent ces sédiments, les mettent en suspension avant de les transporter. Pour modéliser ce phénomène, il est important de quantifier le coefficient de diffusion turbulente des sédiments. Il est habituellement admis que ce coefficient est lié directement à la viscosité turbulente (FREDSOE \& DEIGAARD, 1992 ; LIU \& SATO, 2006 ; SUNTOYO \& TANAKA 2009 ; ABSI 2010). Plusieurs études de modélisation de la concentration des sédiments mis en suspension par des écoulements oscillants ont été effectuées avec différents modèles de turbulence (FREDSOE \& DEIGAARD, 1992 ; LIU \& SATO, 2006 ; SUNTOYO et TANAKA, 2009). Des modèles empiriques de viscosité turbulentes sont utilisés en génie côtier (figures 1). Malgré leur simplicité, ils permettent souvent d'obtenir des résultats intéressants.

Notre étude a pour objectif d'évaluer et de valider certains profils de viscosité turbulente analytiques et/ou empiriques utilisés en génie côtier pour le calcul du transport sédimentaire par comparaison avec la viscosité turbulente moyennée sur une période obtenue à partir d'un modèle à deux équations $k-\omega$.

\section{Profils de viscosité turbulente}

En génie côtier, la viscosité turbulente est obtenue soit à partir de modèles mathématiques souvent issus de la modélisation statistique de la turbulence (modèles à deux équations $k-\varepsilon, k-\omega, \ldots$ ) (FREDSOE \& DEIGAARD, 1992 ; SUNTOYO \& TANAKA, 2009), ou à partir des modèles analytiques ou empiriques (FREDSOE \& DEIGAARD, 1992 ; LIU \& SATO, 2006 ; ABSI, 2010). La figure (1) présente quelques profils de viscosité turbulente dits multicouches.

Parmi les modèles analytiques de viscosité turbulente, deux principaux profils sont largement utilisés en génie côtier. Le premier profil est décrit par une variation parabolique de la viscosité turbulente puis une valeur uniforme, nous l'appellerons le profil parabolique-uniforme (VAN RIJN, 1993 ; LIU \& SATO, 2006). Il est décrit par :

$v_{t}=\left\{\begin{array}{lll}\kappa u_{k} z\left(1-\frac{a}{\delta}\right) & \text { si } & z<\delta / 2 \\ v_{t \max } & \text { st } & z \geqslant \delta / 2\end{array}\right.$

Où $\boldsymbol{\kappa}$ : est la constante de Karman, $u_{z}$ la vitesse de frottement au fond, $z$ la distance à partir du fond et $\delta$ l'épaisseur de la couche limite. Le second profil est donné par une loi exponentiel modulée par une fonction linéaire, on l'appellera le profil exponentiellinéaire (HSU \& JAN, 1998 ; ABSI, 2010). Il est donné par :

$v_{t}-\operatorname{aru} u_{k} \operatorname{xexp}\left(-C_{A} \frac{g}{\delta}\right)$ 
Où $\alpha$ et $C_{A}$ sont deux coefficients.

Les équations (1) et (2) s'écrivent sous forme adimensionnelle :

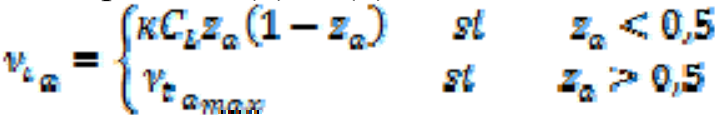

et :

$v_{t_{n}}=\alpha \kappa z_{\alpha} \exp \left(-C_{\mathrm{d}} z_{\alpha}\right)$

Avec $v_{t_{\mathrm{rm}}}=v_{\mathrm{z}} /\left(u_{+} \delta\right) ; z_{\mathrm{m}}=z / \delta$ et $v_{\tau_{\alpha_{\max }}}=\kappa C_{L} z_{\alpha_{\max }}\left(1-z_{\alpha_{\max }}\right)$

Dans l'équation (3), nous avons introduit un coefficient d'ajustement $C_{L}$. Le profil initial donné par l'équation (1) ne contient pas ce coefficient $\left(C_{L}=1\right)$.

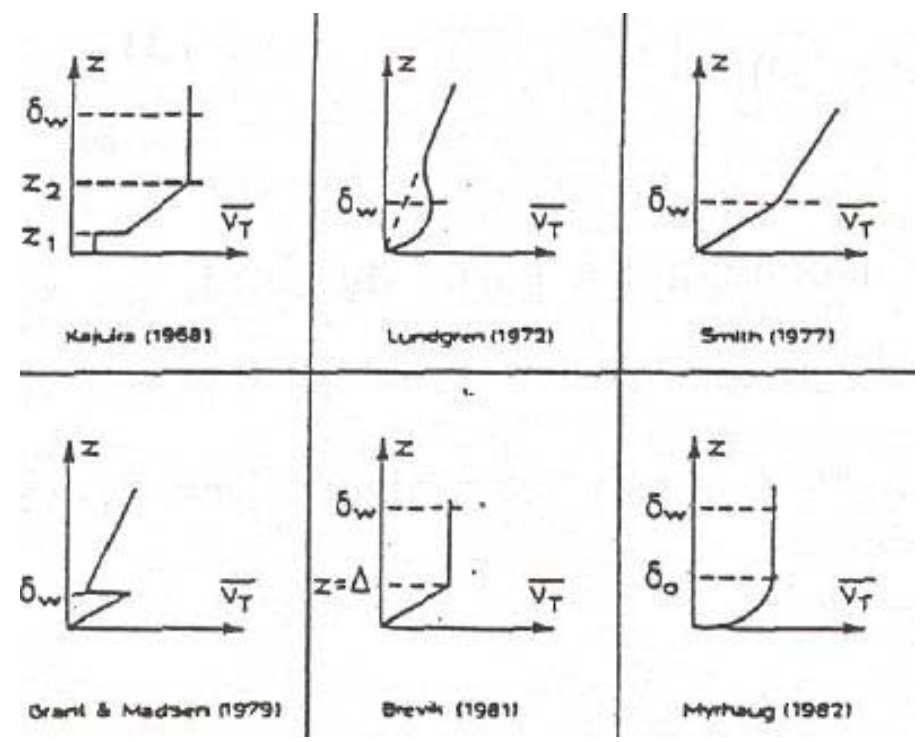

Figure 1. Modèles empiriques de viscosité turbulente pour la couche limite sur un fond marin (FREDSOE \& DEIGAARD, 1992).

\section{Modèle à deux équations $\boldsymbol{k}$ - $\boldsymbol{\omega}$}

Nous utilisons le modèle $k-\omega$ BSL (Baseline) de MENTER (1994). Les équations de l'énergie cinétique turbulente $k$, de sa dissipation $\omega$ ainsi que la viscosité turbulente sont données par

$$
\begin{aligned}
& \frac{\partial k}{\partial t}=\frac{\partial}{\partial z}\left\{\left(v+v_{t} \sigma_{h \omega}\right) \frac{\partial k}{\partial z}\right\}+v_{t}\left(\frac{\partial u}{\partial z}\right)^{2}-\beta^{*} \omega k, \\
& \frac{\partial \omega}{\partial t}=\frac{\partial}{\partial z}\left\{\left(v+v_{t} \sigma_{\omega}\right) \frac{\partial \omega}{\partial z}\right\}+\gamma\left(\frac{\partial u}{\partial z}\right)^{2}-\beta \omega^{2}+2\left(1-F_{1}\right) \sigma_{\omega 2} \frac{1}{\omega} \frac{\partial k}{\partial z} \frac{\partial \omega}{\partial z} \\
& v_{t}=\frac{k}{\omega},
\end{aligned}
$$


Les constantes du modèle sont données par $\sigma_{k \omega}=0,5, \beta^{*}=0,09, \sigma_{\omega}=0,5, \gamma=0,553$ et $\beta=0,075$ (SUNTOYO \& TANAKA, 2009).

C'est un modèle à deux équations qui donne des résultats similaires à ceux du modèle $k-\omega$ de WILCOX (1988) dans la partie basse de la couche limite et change progressivement vers le modèle $k-\varepsilon$ de JONES et LAUNDER (1972) dans la partie externe de la couche limite. Le raccordement entre les deux régions est assuré par la fonction $\vec{F}_{1}$.

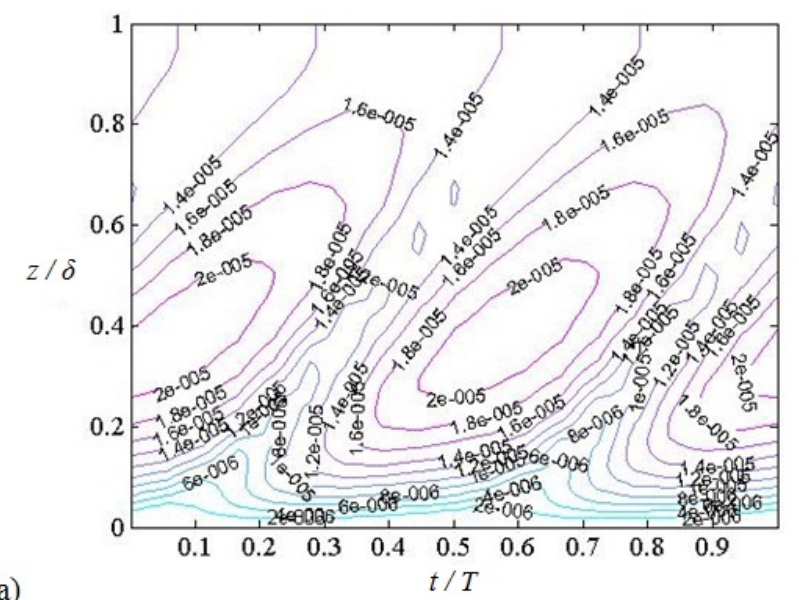

(a)

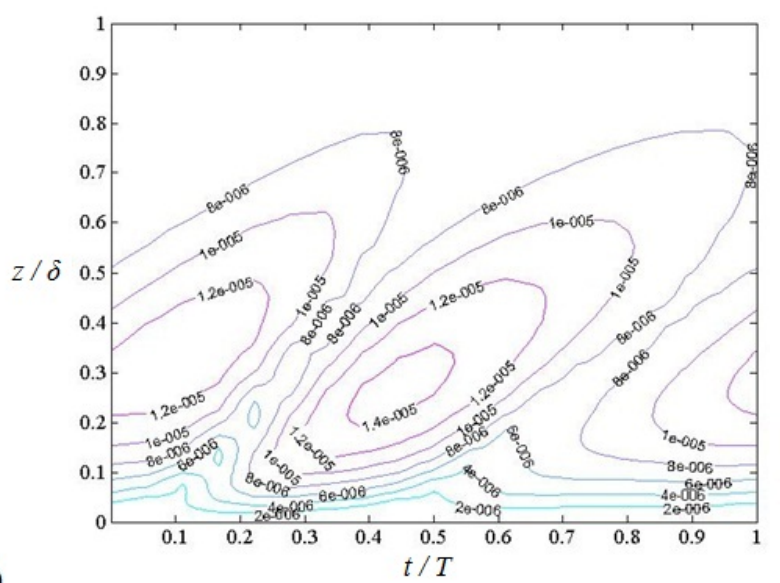

(b)

Figure 2. Variation temporelle et spatiale de la viscosité turbulente pendant une période de houle obtenue avec le modèle à deux équations $k$ - $\omega$; (a) houle sinusoïdale $(N i=0,5)$;

(b) houle asymétrique $(\mathrm{Ni}=0,67)$ (ABSI \& TANAKA, 2011).

La figure (2) montre la variation spatiale et temporelle de la viscosité turbulente sur une période de houle $T$ obtenue avec le modèle BSL $k$ - $\omega$. Les conditions de l'écoulement sont $U_{0}=3,63 \mathrm{~m} / \mathrm{s} ; a_{m}=1,73 \mathrm{~m} ; T=3 \mathrm{~s} ; k_{s}=1,5 \mathrm{~cm}, R e=437000$. La viscosité turbulente est fortement instationnaire pour les deux cas : houle sinusoïdale donnée par une vitesse à l'extérieur de la couche limite $U(b)-U_{7} \operatorname{sirc}(\omega)$ ) (figure 2.a) et houle asymétrique donnée par $U(t)=U_{1} \sin (\omega t)-U_{2} \cos (2 \omega t)$ (figure 2.b). Pour la houle asymétrique, 


\section{XII ${ }^{\text {èmes }}$ Journées Nationales Génie Côtier - Génie Civil \\ Cherbourg, 12-14 juin 2012}

l'asymétrie est donnée par la degré d'asymétrie (ou le paramètre de non linéarité) $N_{i}=\left(U_{1}+U_{i}\right) /\left(2 U_{1}\right)=0,67$ (voir ABSI et TANAKA, 2011). Cependant il est possible de décrire la viscosité turbulente moyennée sur la période par le profil analytique exponentiel-linéaire (figure 3) donné par l'équation (2).

(a)

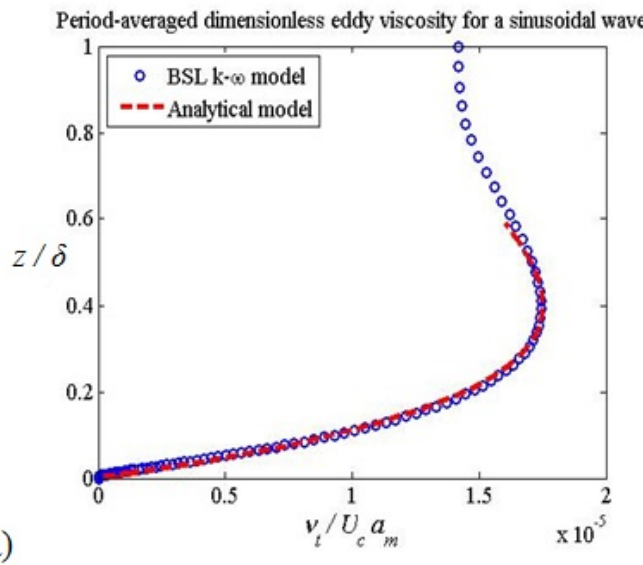

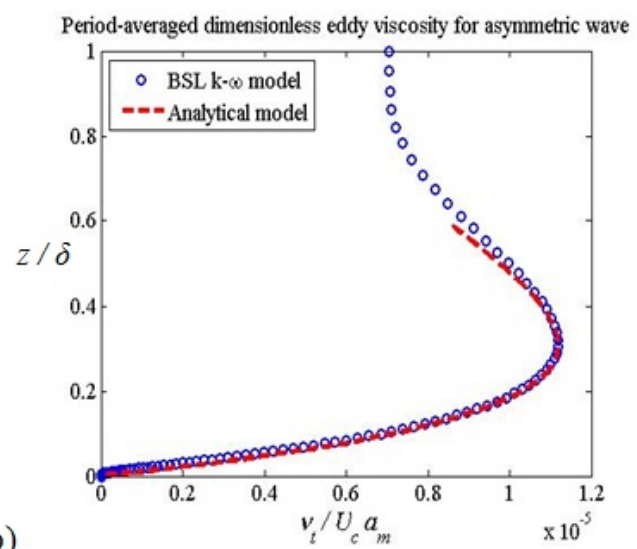

(b)

Figure 3. Viscosité turbulente moyennée sur la période de la houle; (a) houle sinusoïdale $(N i=0,5)$; (b) houle asymétrique $(N i=0,67)$. Symboles : résultats $d u$ modèles $k$ - $\omega$, courbes : profil analytique exponentiel-linéaire (équation 2).

\section{Validation des deux profils de viscosité turbulente}

La figure 4 montre que le profil parabolique-uniforme permet une bonne description de la viscosité turbulente pour des valeurs de $\omega_{m} i_{2} \geq 500$.

La figure 5 montre que le profil exponentiel-linéaire permet une bonne description de la viscosité turbulente pour des valeurs de $a_{m} / k_{g}<500$ sur une hauteur allant jusqu'à $z_{\alpha} \simeq 0,8$.

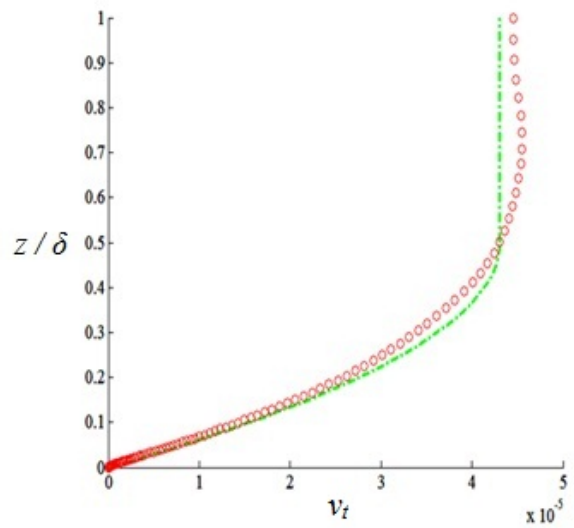

(a) $a_{m} / k_{s}=500$

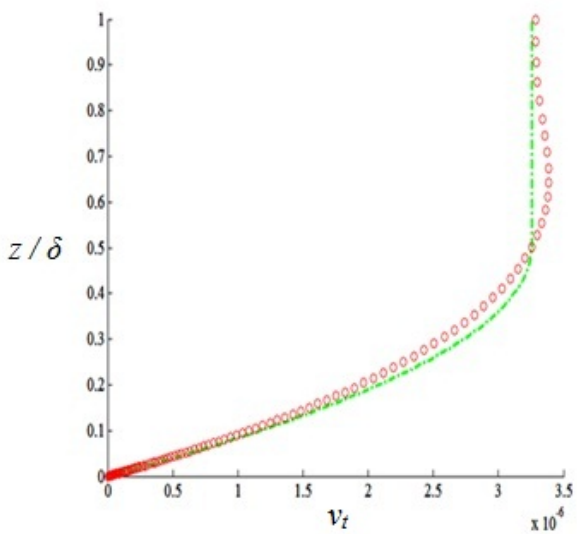

(b) $a_{m} / k_{s}=5000$

Figure 4. Profils de viscosité turbulente décrits par l'équation (1). Symboles : résultats du modèles $k-\omega$, courbes : profil analytique parabolique-uniforme (équation 1). 


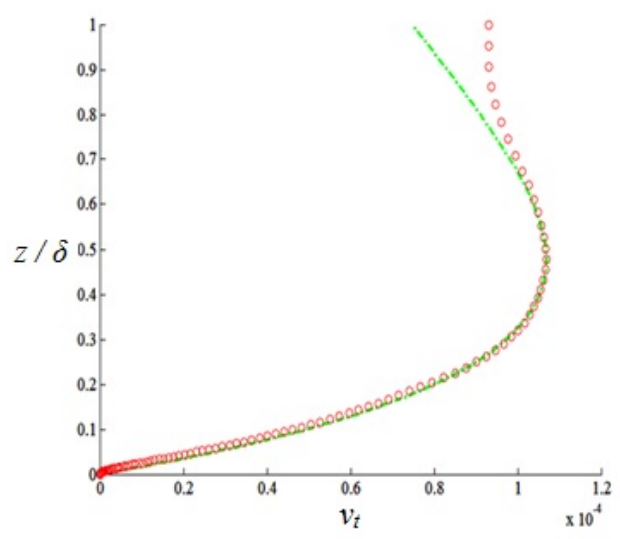

(a) $a_{m} / k_{s}=150$

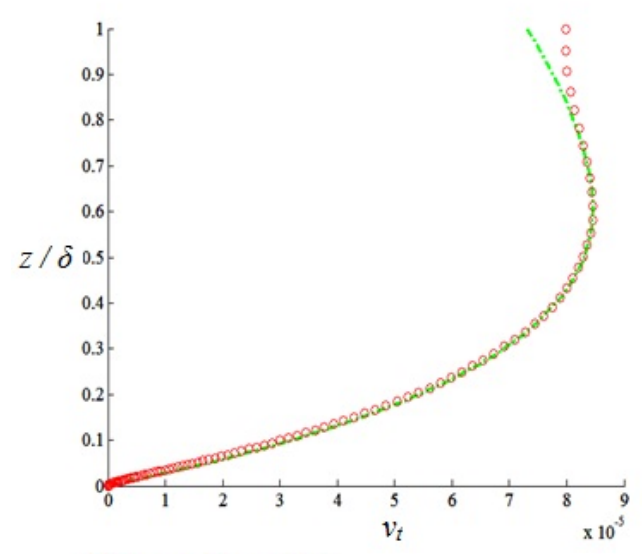

(d) $a_{m} / k_{s}=250$

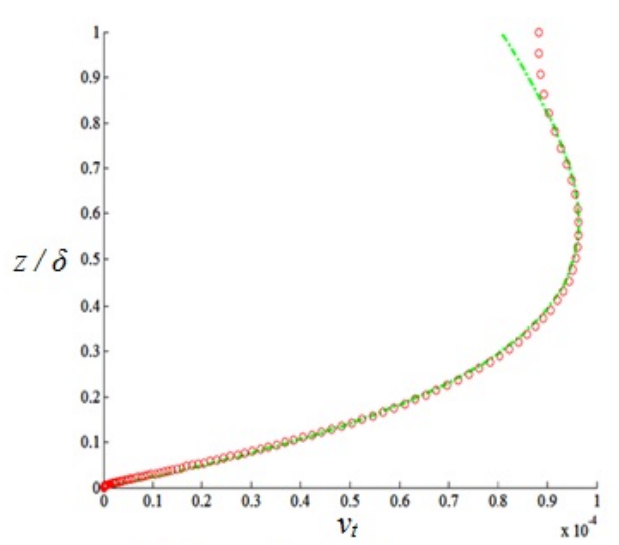

(b) $a_{m} / k_{s}=200$

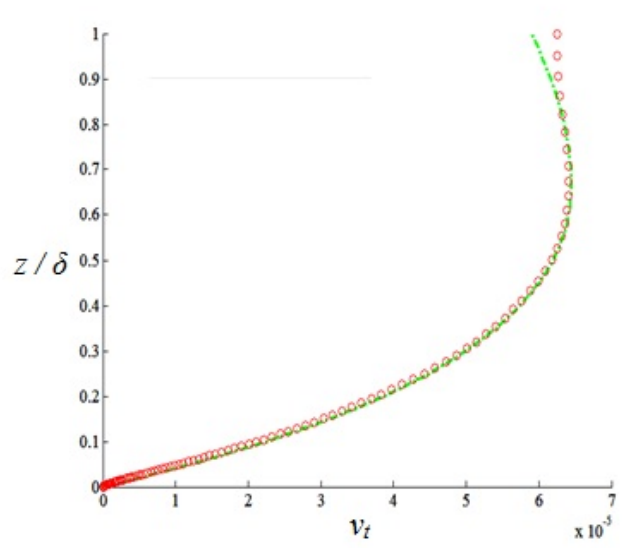

(d) $a_{m} / k_{s}=350$

Figure 5. Profils de viscosité turbulente décrits par l'équation (2). Symboles : résultats du modèles $k-\omega$, courbes : profil analytique exponentiel-linéaire (équation 2).

\section{Calibration des deux profils de viscosité turbulente}

L'étude montre que les coefficients des deux profils de viscosité turbulente (parabolique-uniforme et exponentiel-linéaire) ne sont pas des constantes et qu'ils doivent varier en fonction du paramètre $a_{m} / k_{g}$.

L'analyse (figure 6), permet de déduire des courbes de tendance décrivant la variation de ces coefficients en fonction du paramètre $a_{m} / k_{g}$. Nous proposons les équations suivantes :

$C_{L_{n}}=0,4\left(a_{m g} / k_{g}\right)^{-: 1}$

$\alpha=0,2\left(a_{m} / k_{s}\right)-0.97$

$c_{A}-2 g_{p} z\left(a_{m} / k_{s}\right)^{-0.82}$

Les équations (6) et (7) permettent une très bonne description des coefficients d'ajustements $r_{\mathrm{L}}$ et $\alpha$ (figures 6.a et 6.b) respectivement pour les profils paraboliqueuniforme (équation 1) et exponentiel-linéaire (équation 2). Ce qui implique une très 


\section{XII ${ }^{\text {èmes }}$ Journées Nationales Génie Côtier - Génie Civil \\ Cherbourg, 12-14 juin 2012}

bonne prévision de la valeur maximale de la viscosité turbulente. La courbe relative au coefficient $C_{A}$ est de moins bonne qualité $\left(\mathrm{R}^{2}=0,95\right)$. Ce coefficient est lié à la prévision de la position de la viscosité turbulente maximale obtenue à partir du profil exponentiellinéaire (équation 2).

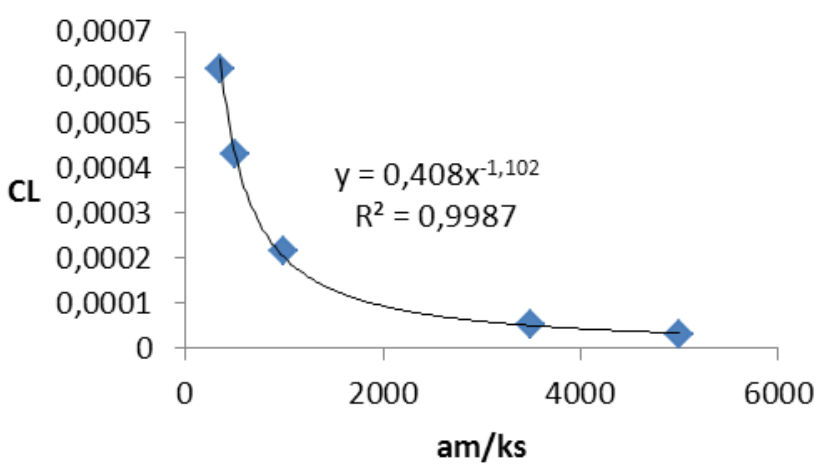

(a)

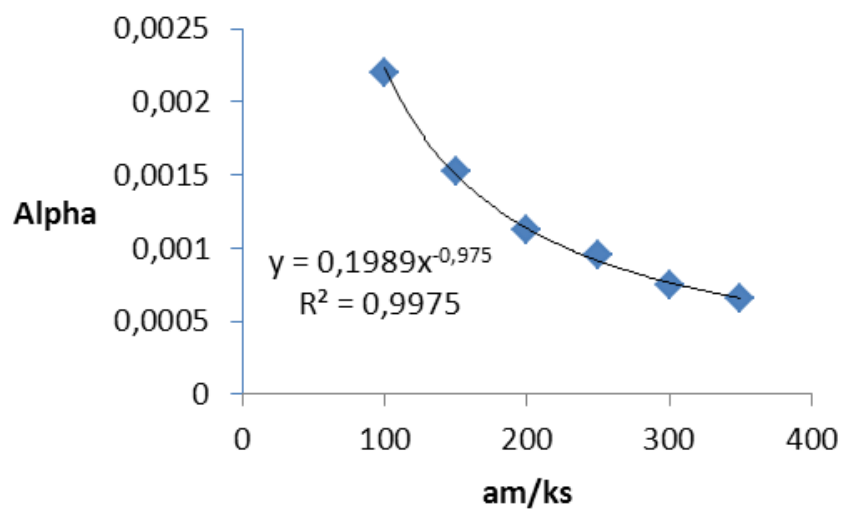

(b)

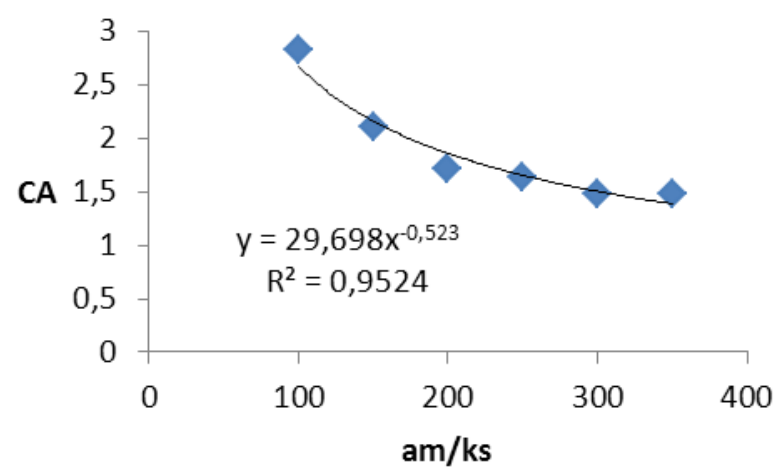

(c)

Figure 6. Calibration des paramètres des deux profils de viscosité turbulente.

\section{Conclusions}

Dans le cadre de notre étude, des comparaisons entre les résultats numériques du modèle à deux équations $k$ - $\omega$ et les deux profils analytiques de viscosité turbulente ont 
été réalisées pour différentes valeurs du paramètre $a_{m} / k_{g}$. Les résultats ont montré que :

- Le profil parabolique-uniforme (équation 1) semble décrire correctement les données du modèle $k-\omega$ pour $a_{m} / k_{s} \geq 500$, alors que le profil exponentiel-linéaire (équation

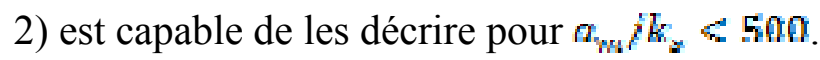

- Le profil parabolique-uniforme nécessite un coefficient d'ajustement $C_{L}$ qui dépend du paramètre $a_{\mathrm{m}} / \tilde{k}_{\mathrm{z}}$

- Des expressions ont été proposées pour décrire la variation des différents coefficients des deux profils en fonction du paramètre $a_{w_{s}} / k_{\mathrm{s}}$

\section{Remerciements}

Une partie de cette étude a été financée par la Société Japonaise de la Promotion de la Science (JSPS) dans le cadre du programme de recherche au Japon "FY2010 JSPS Invitation Fellowship Program for Research in Japan" (No. S-10168).

\section{Références bibliographiques}

ABSI R. (2010). Concentration profiles for fine and coarse sediments suspended by waves over ripples: An analytical study with the 1-DV gradient diffusion model. Advances in Water Resources, Vol. 33, pp 411-418. doi:10.1016/j.advwatres.2010.01.006

ABSI R., TANAKA H. (2011). Toward improving prediction of sediment transport over wave-induced ripples, Tohoku Journal of Natural Disaster Science, Vol. 47, pp 213-218.

FREDSOE J., DEIGAARD R. (1992). Mechanics of coastal sediment transport, World Scientific, 369 p. doi:10.1142/9789812385314

HSU T.W., JAN C.D. (1998). Calibration of BusingerArya type of eddy viscosity models parameters. J. Waterway Port Coastal Ocean Eng., ASCE, Vol. 124(5), pp 281-284. doi:10.1061/(ASCE)0733-950X(1998)124:5(281)

JONES W.P., LAUNDER B.E. (1972). The prediction of laminarization with a twoequation model of turbulence. International Journal of Heat Mass Transfer 15, pp 301-314. doi:10.1016/0017-9310(72)90076-2

LIU H., SATO S. (2006). A two-phase flow model for asymmetric sheetflow conditions. Coastal Engineering, Vol. 53, pp 825-843. doi:10.1016/j.coastaleng.2006.04.002

MENTER F.R. (1994). Two-equation eddy-viscosity turbulence models for engineering applications. AIAA Journal 32 (8), pp 1598-1605. doi:10.2514/3.12149

SUNTOYO, TANAKA H. (2009). Effect of bed roughness on turbulent boundary layer and net sediment transport under asymmetric waves. Coastal Engineering, 56(9), pp 960-969. doi:10.1016/j.coastaleng.2009.06.005

VAN RIJN L.C. (1993). Principles of sediment transport in River, Estuaries and Coastal Seas. Aqua, Amsterdam.

WILCOX D.C. (1988). Reassessment of the scale-determining equation for advanced turbulent models. AIAA Journal, Vol. 26 (11), pp 1299-1310. doi:10.2514/3.10041 